\title{
Revisión de las definiciones de proposición y enunciado en su relación con las matemáticas
}

\author{
Review of the Definitions of Propositions and Statement in their Relationship with Mathematics \\ Revisão das definições de proposição e enunciado em sua relação com as matemáticas
}

Luis Hurtado Mondoñedo (http://orcid.org/0000-0001-5373-556X)

Departamento de Ciencias, Universidad de Ingeniería y Tecnología (UTEC), Lima, Perú
Recibido: 26-07-16 Revisado: 06-12-16 Aceptado: 31-01-17

Publicado: 05-05-17
RESUMEN. En matemáticas trabajamos con proposiciones. El término proposición es tomado de la lógica y suele ser definido como un enunciado que puede ser calificado de verdadero o falso. Se considera la proposición como un enunciado y este último como una frase u oración. Según lo anterior, por ejemplo, la expresión $3<7$, que es claramente una proposición verdadera del campo matemático, no lo sería si atendemos a lo dicho líneas arriba. En el presente artículo se hace una revisión de las definiciones de lógica, proposición y enunciado tomadas en una muestra de textos de secundaria y universitarios que tratan el tema y, a partir de ello, se analiza la coherencia de las definiciones de proposición y enunciado, así como su pertinencia en relación con las matemáticas.
Palabras clave: enunciado, proposición, lógica, matemáticas, textos escolares y universitarios
ABSTRACT. In mathematics we work with propositions. The term proposition is taken from logic and is usually defined as a statement that can be qualified as true or false. A proposition is considered as a formulation, and this is a phrase or sentence. According to the above, for example, the expression $3<7$, which is clearly a true statement from the field of mathematics, would not be such according to what was expressed above. This article reviews the definitions of logic, proposition and statement taken in a sample of high school and college texts that deal with the subject and, on that basis, the consistency of the definitions of proposition and statement are analyzed, as well as their relevance in relation to mathematics.
Keywords: statement, proposition, logic, mathematics, high school and college text 
RESUMO. Nas matemáticas trabalhamos com proposições. O termo proposição vem da lógica e costuma ser definido como um enunciado que pode ser qualificado de verdadeiro ou falso. Considera-se a proposição como um enunciado e este como uma frase ou oração. Segundo o parágrafo anterior, por exemplo, a expressão $3<7$, que é claramente uma proposição verdadeira na área das matemáticas, não seria tal se atendêssemos ao mencionado acima. Neste artigo, faz-se uma

Palavras-chave: enunciado, proposição, lógica, matemáticas, textos do ensino médio e superior revisão das definições de lógica, proposição e enunciado, extraídas de uma amostra de textos do ensino médio e superior que tratam o tema e, a partir daí, analisa-se a coerência das definições de proposição e enunciado, assim como a sua pertinência com relação às matemáticas.

La matemática es una ciencia formal. Partiendo de axiomas y definiciones se formula una serie de proposiciones que, expresadas en lenguaje simbólico y siguiendo reglas de inferencia, permiten validarlas y construir todo un cuerpo de conocimientos. Los axiomas y las definiciones son convenciones. Un axioma enuncia en forma clara y breve verdades matemáticas que, aunque no siempre nos resultan evidentes, son aceptadas sin demostración. Según Young (1929) se les considera como proposiciones aceptadas sin demostración para servir de base al razonamiento que se va a desarrollar. Es claro que en las matemáticas trabajamos con proposiciones y eso nos lleva a dejar explícita su definición. La definición de cierto término o concepto matemático hace claro el significado preciso que se le debe atribuir.

Proposición suele definirse como un enunciado que puede ser calificado de verdadero o falso. Una de las reglas de Pascal para las definiciones propone que “al definir, no emplear otra clase de términos que los que sean perfectamente claros por sí mismos o que ya hayan sido definidos" (Pascal, como se citó en Young, 1929, p.174). En tanto proposición ha sido definido como un enunciado, entonces es necesario hacer claro el significado que se le atribuirá a este último término.

Un enunciado es considerado como una frase u oración. De aquí que, atendiendo a la regla de Pascal, tenemos que o bien el término oración es perfectamente claro por sí mismo o debemos definirlo. Una revisión de los textos muestra que se toma oración como un término perfectamente claro. Esto es como una estructura gramatical formada por la unión de un sujeto y un predicado?.

El término proposición es tomado de la lógica. En los textos de lógica las proposiciones simples suelen ser expresadas como oraciones que o bien son verdaderas o bien son falsas. Asociar el término proposición con una oración no resulta pertinente en el trabajo con expresiones matemáticas. Así por ejemplo la expresión $3<7$ no es una oración y por tanto, si atendemos a lo dicho líneas arriba, la expresión no sería una proposición. Sin embargo $3<7$ enuncia claramente una proposición verdadera del campo matemático. Por lo mencionado se hace necesario revisar y analizar las definiciones de 
proposición y enunciado en su relación con las matemáticas. Dado que la construcción del cuerpo de conocimientos matemáticos se hace formulando proposiciones, entonces la definición del término proposición debe ser pertinente con las matemáticas. De aquí la importancia del presente estudio. En este trabajo se hace una revisión de las definiciones de lógica, proposición y enunciado tomadas en una muestra de textos de la secundaria y universitarios que tratan el tema.

\section{LÓGICA Y MATEMÁTICA}

“La lógica y la matemática, por ocuparse de inventar entes formales y de establecer relaciones entre ellos, se llaman a menudo ciencias formales" (Bunge, 1966, p.7). Son ciencias deductivas en las que sus demostraciones se basan en un conjunto de definiciones y reglas propias. Por su calidad de ciencias formales la matemática y la lógica guardan estrecha relación. Los conceptos y principios materia de la lógica examinan los mecanismos del pensamiento racional, de aquí que su comprensión facilita el aprendizaje de las matemáticas. Pero no solo de las matemáticas. La enseñanza de la lógica “habilita para el manejo adecuado del lenguaje de la ciencia y como método para construir la ciencia” (Vásquez, 2013, p.155). La matemática y la lógica son consideradas áreas importantes en la educación. Sin embargo, mientras que el estudio de los temas de matemáticas se ha mantenido en los programas escolares, los temas de lógica han ido diluyéndose.

Actualmente en los documentos oficiales de la educación básica regular no figura el estudio de la lógica como un área independiente. Hasta el Diseño Curricular Nacional (DCN) del 2009, que rigió por varios años después, se incluía en el área de matemática conocimientos de relaciones lógicas y conjuntos. En la tercera versión del marco del sistema curricular nacional (MCN), dentro de la descripción de la competencia actúa matemáticamente en diversos contextos, se dice que las matemáticas ayudan a enfrentar y asumir de manera razonada y lógica los problemas que el mundo nos presenta. Se destaca el razonamiento lógico y crítico en tanto este "favorece la coherencia lógica de las ideas ... así como hacer deducciones o inferencias" (MINEDU, 2014, p.74). Todo indica que solo con el aprendizaje de la matemática se buscaría desarrollar este razonamiento. No son pocas las voces que, en su momento, se levantaron por quitar la asignatura de Filosofía, y con ello la lógica, del programa oficial de la educación básica ${ }^{2}$. Entre otras críticas se advertía que el diseño curricular no daba lugar a la lógica que permite a los estudiantes ejercitarse en el pensamiento riguroso.

Pero también se dieron voces contrarias. Boisvert (2004) señala que "es cierto que la lógica es útil para la formación y el ejercicio del pensamiento crítico, pero no es la única herramienta existente" (p.62). En 1948 la educación básica incluía el estudio de la lógica. Ese año, Miró Quesada, destacado investigador peruano que se preocupó por introducir la lógica en el Perú, publica Lógica curso escolar, donde señala que su texto se sujeta al programa oficial. Más tarde, en 1961, los temas de lógica pasan a formar parte del curso de Filosofía. Ahí se mantuvo hasta el 2002, año en que fue retirado del programa de estudios escolares. En la educación superior, a pesar de la importancia que tiene para la investigación científica, el interés por la lógica también se ha visto debilitado. En su estudio acerca de los textos de lógica, Vásquez (2013) refiere que “la enseñanza-aprendizaje de la

2 En el 2004, dos años después de la medida gubernamental de suprimir la asignatura de Filosofía, la comunidad filosófica peruana se manifestó abiertamente a favor de su restablecimiento en las escuelas. 
lógica general en el nivel universitario, de 1940 hasta la actualidad, muestra una disminución de su importancia en la formación profesional" (p.153).

En la educación básica, dentro de los temas de número, relaciones y funciones del área de matemática se incluían conocimientos de relaciones lógicas y conjuntos. Estos, a su vez incluían, lo referido a enunciado y proposición. Su estudio se iniciaba en el segundo grado de secundaria y continuaba hasta el quinto grado. De aquí que este tema sea tratado en los libros de texto de mayor demanda publicados por las principales editoriales en nuestro país. En la educación superior los temas de lógica también suelen incluirse en el primer curso de matemática de estudios generales 0 formación transversal. Incluir temas de lógica dentro del silabo de un curso de matemática supone que los contenidos ahí tratados contribuirán al aprendizaje de las matemáticas. Se esperaría que los conceptos, reglas y ejemplos guarden relación con los temas propios de la matemática. Mayor razón si los temas de lógica son los primeros que se estudian en el curso. Por lo general después del capítulo de lógica le sigue el que trata los temas de conjuntos. Esto queda justificado, ya que la teoría de conjuntos puede construirse usando conectores lógicos y cuantificadores. Para los siguientes temas matemáticos, se justificaría debido a que muchos teoremas son enunciados según la forma "Si .... entonces ...". Su demostración requiere de la lógica para la solidez de su argumentación.

Para el presente trabajo se tomó una muestra intencional de textos de matemática y lógica en los que se abordan temas propios de la lógica. La muestra incluyó siete textos de secundaria de las editoriales más reconocidas del país y nueve textos universitarios. La revisión de los textos se centró en los siguientes aspectos:
a) Análisis de la definición de lógica, proposición y enunciado.
b) Coherencia de la definición de enunciado respecto a la de proposición.
c) Coherencia de la definición de enunciado y proposición respecto de las matemáticas.

\section{DEFINICIÓN DE LÓGICA EN LOS TEXTOS}

Lógica suele ser el primer tema tratado en los libros de texto de matemática del segundo al quinto de secundaria. En los textos del primer curso de matemática de la educación superior los temas de lógica suelen presentarse en el primer capítulo. Encontramos distintas definiciones o descripciones para referirse a ella.

\section{Definiciones tomadas de textos de secundaria:}

L1. La disciplina que determina por medio de reglas y técnicas la validez de un argumento se denomina Lógica (Grupo Editorial Norma, 2009a, p.12).

L2. Lógica es la disciplina que ayuda a determinar si los razonamientos son válidos o no. Parte de un conjunto de informaciones para deducir otra. La información inicial recibe el nombre de premisa y para el análisis se considera verdadera, la que se deduce recibe el nombre de conclusión (Grupo Editorial Norma, 2009b, p.12).

L3. La ciencia que estudia los principios formales del conocimiento, es decir, aquellas condiciones que deben cumplirse para que un enunciado, cualquiera que sea su contenido, pueda considerarse como verdadero, y no solo como una hipótesis, se denomina lógica (Grupo Editorial Norma, 2009c, p.12). 
L4. Es la lógica, la ciencia que permitirá aprender a razonar de manera correcta (Doroteo \& Gálvez, 2005, p.12).

L5. La lógica es la ciencia que estudia los métodos y procedimientos que determinan la validez o invalidez de las inferencias o argumentos. Inferir es razonar para obtener una conclusión de un conjunto de afirmaciones que llamamos premisas (Editorial Santillana, 2009, p.18).

\section{Definiciones tomadas de textos universitarios:}

L6. Ciencia que estudia los métodos o procedimientos que aplican definiciones y leyes o reglas con el propósito de determinar la validez o invalidez de las inferencias (Rosales, 1994, p.15).

L7. La Lógica es el estudio de los métodos y principios usados para distinguir el razonamiento correcto del incorrecto (Copi, 1964, p.17).

L8. La lógica es la ciencia y a la vez el arte del estudio de los razonamientos o inferencias (Katayama, 2003, p.19).

De las definiciones anteriores podemos observar que la lógica es considerada como una disciplina (L1, L2), como una ciencia (L3, L4, L5, L6, L8) y como un arte (L8). La lógica y la matemática son consideradas ciencias formales. Palacios y García (1998) señalan que la ciencia puede ser definida como un sistema de proposiciones y que las ciencias formales "establecen la verdad de sus proposiciones mediante deducciones o demostraciones" (p.61). Miró Quesada (1978) define la lógica como "la teoría de la deducción" (p.129) ${ }^{3}$. Se trata de una teoría científica, ya que comprende un conjunto de conocimientos sistemáticamente enlazados que versan sobre un campo de estudio bien definido que, en el caso de la lógica, es la deducción. Miró Quesada señala que, entre otras cosas, cuando existe un método que permite justificar y aumentar los conocimientos con seguridad, se trata de una teoría científica. En L6 Rosales (1994) parece querer destacar esta característica al decir que la lógica, con el propósito de determinar lo válido o no de una inferencia, estudia los métodos o procedimientos que aplican sobre las definiciones y reglas dadas. Lo mismo parece ocurrir en L7 cuando Copi (1964) refiere a la lógica como el estudio de los métodos y principios.

Aunque en la definición L7 no lo indica, Vásquez (2013) refiere que en su Introducción a la Lógica, Copi explicita como uno de los objetivos "estudiar la lógica como un arte y como ciencia, para ello debe

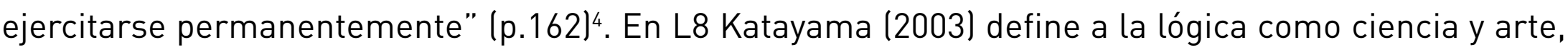
pero no explica por qué la considera de este modo ${ }^{5}$. Podemos encontrar en Aristóteles referencias del considerar la lógica como un arte. Para Aristóteles, considerado el padre de la lógica, arte y ciencia son formas distintas de conocimiento. El primero es un saber técnico cuyo fin es productivo mientras que el segundo no tiene ningún fin específico y constituye un saber teórico que estudia las causas y los principios de las cosas. En su Metafísica Aristóteles (2007) dice que, a diferencia de los demás animales, “el género humano posee, para conducirse, el arte y el razonamiento" (p.7). Santo Tomás (como se citó en Moreno, 1971) en sus Comentarios a los Segundos Analíticos escribe "es necesario entonces un

\footnotetext{
3 La definición es tomada de la parte correspondiente a Lógica escrita por Francisco Miró Quesada del texto para el 5to año de secundaria Introducción a la filosofía y lógica. El texto fusiona los libros Introducción a la Filosofía de Augusto Salazar Bondy (1925-1974) y Lógica de Miró Quesada. Este último, posterior a la muerte de Salazar, reajusta los temas e introduce otros para adaptarlos al programa oficial vigente a 1978.

4 La definición de Copi es frecuentemente citada. Vásquez refiere que su libro ha sido la base en la formación de muchos profesores que enseñan lógica en el nivel universitario actual.

5 En la introducción de su libro Katayama señala que el texto tiene por objeto realizar una presentación panorámica de las principales aplicaciones de la lógica.
} 
arte que dirija el acto mismo de la razón ... Este arte es la lógica, esto es, la ciencia racional" (p.4). La lógica era interpretada como el arte de las artes, ya que sería ella quien dirija el acto de la razón.

La revisión de los ocho textos citados ha evidenciado que no existe univocidad tanto en la definición de lógica como en la descripción de su objeto de estudio. Esto se puede explicar debido a que una definición no es definitiva. Se trata de una convención del significado atribuido durante el tiempo que permanezca vigente. La extensión y el sentido del término están limitados por el alcance del grupo al cual se dirige la definición. Según Young (1929) "el alcance del punto de vista del alumno es lo que debe controlar la definición, no la del maestro" (p. 178). En tanto se va ensanchando el horizonte del estudiante resulta razonable que las definiciones sean ampliadas al avanzar en la secundaria y, sobre todo, al pasar a la etapa universitaria. Sin embargo nuestra revisión muestra que las definiciones dadas en los textos de secundaria no avanzan hacia una generalización. Al mismo tiempo, textos de diferentes grados de una misma editorial no adoptan una misma definición de lógica siendo la dada en el grado inferior (L1) más general que la del grado superior (L3). La descripción del objeto de estudio de la lógica refiere a términos como inferencia, argumento y razonamiento. Por lo general estos términos no son definidos o no se da una noción de ellos.

\section{RAZONAMIENTO, INFERENCIA Y ARGUMENTO}

En las definiciones citadas vemos que, al describir el objeto de estudio de la lógica, se alude a distintos términos como razonamiento (L2, L4, L7, L8), inferencia (L5, L6, L8), argumento (L1, L5) y enunciado (L3). Pero en los textos, de donde estas definiciones son tomadas, no siempre se precisa el significado de los términos que dichas definiciones refieren. En L8 Katayama (2003) toma los términos inferencia y razonamiento como sinónimos. En su texto no hace ninguna distinción entre ellos ni tampoco los define. Sin embargo describe argumento como un tipo de razonamiento o inferencia que se caracteriza por sostener una conclusión apoyada por premisas. L7 también es citada, junto a otras definiciones, por Moreno (1971) en su Lógica Matemáticab. No precisa el significado del término razonamiento, pero indica que la lógica “investiga cuestiones vinculadas con la corrección o incorrección de las argumentaciones" (Moreno, 1971, p.3). Tampoco llega a definir argumento pero inicialmente menciona que el problema central de la lógica es establecer las condiciones por las cuales "un enunciado puede ser considerado como conclusión derivada de otros enunciados llamados premisas" (Moreno, 1971, p.1). En otro texto del mismo autor encontramos que "todo sistema de premisas y conclusión es llamado argumentación" (Moreno, 1967, p.13). Esta misma definición es adoptada por Bustamante (2009) quien, para conseguir el propósito de distinguir el razonamiento correcto del incorrecto empieza por considerar los elementos del lenguaje usados para expresar estos razonamientos. En la definición L6 Rosales habla de inferencias y este define la inferencia como "un proceso que consiste en obtener la conclusión a partir de un conjunto de premisas" (Rosales, 1994, p.15).

Como mencionamos anteriormente, para Miró Quesada (1978) la lógica es la teoría de la deducción la cual consiste en pasar de la verdad de las premisas a la verdad de la conclusión. Habla de deducción y no de inferencias o razonamiento. Hace la distinción entre ellos pero anota que usará los términos 
deducción e inferencia como sinónimos. Distingue deducción de inferencia debido a que esta última puede significar también inducción, que es un tipo de conocimiento diferente al deductivo que es propio de la lógica. Distingue deducción de razonamiento ya que este sería todo tipo de pensamiento que trata de justificar racionalmente una tesis. De este modo considera que una justificación por analogía, una inferencia inductiva, una verificación experimental o la invalidación de una hipótesis por un contraejemplo son también razonamientos.

\section{DEFINICIÓN DE PROPOSICIÓN EN LOS TEXTOS}

En la definición de lógica se hace referencia a términos como razonamientos, argumentos, inferencias y deducciones. La revisión muestra que, básicamente, se les otorga el mismo sentido cuando se señala el propósito de la lógica. También vemos que todas ellas hablan de premisas y de conclusión. Para Bustamante (2009) “un argumento o razonamiento es un bloque de proposiciones con el cual se afirma que una de ellas, llamada conclusión, se deriva, se desprende o se sigue como consecuencia de otras proposiciones del mismo bloque llamadas premisas" (p.11). La mayoría de autores consideran la premisa y la conclusión como proposiciones. A continuación mencionaremos diferentes definiciones o descripciones de proposición.

\section{Definiciones en los textos de secundaria:}

P1. Tiene un carácter aseverativo, pues afirma o niega algo sobre un sujeto conocido. Una expresión puede ser comprobada o refutada, lo que le da un valor de verdad por el cual puede ser verdadera o falsa, pero no ambas cosas (Grupo Editorial Norma, 2009a, p.12).

P2. A todo enunciado coherente que se caracteriza por el hecho de poseer un valor de verdad, verdadero o falso, sin ambigüedad, en un determinado contexto, se le llama proposición (Grupo Editorial Norma, 2009c, p.12).

P3. Son enunciados cuyo significado puede calificarse como verdadero o falso. Formalmente se dice que una proposición tiene un único valor de verdad: verdadero o falso, pero no ambos (Grupo Editorial Norma, 2009d, p.12).

P4. Una proposición lógica es todo enunciado que tiene un valor de verdad: verdadero (V) o falso (F), pero no ambos a la vez (Doroteo \& Gálvez, 2005, p.12).

P5. Las proposiciones son expresiones del lenguaje que pueden clasificarse como verdaderas o falsas. Las preguntas, órdenes o exclamaciones no son proposiciones (Editorial Santillana, 2009, p.18).

P6. Es un enunciado que tiene valor de verdad (verdadero o falso) (Editorial Santillana, 2012, p.11).

P7. La proposición es la expresión del lenguaje cotidiano que admite la posibilidad de ser verdadera o falsa, pero que no puede ser verdadera y falsa a la vez (Coveñas, 2012, p.9).

\section{Definiciones en los textos universitarios:}

P8. Una proposición es un enunciado que puede ser calificado o bien como verdadero o bien como falso, pero no ambos a la vez (Curo \& Martínez, 2013, p.13).

P9. Una proposición es un enunciado que puede ser verdadero o falso (Zúñiga, 2013, p.1).

P10. Las proposiciones son expresiones del lenguaje de las que tiene sentido decir que son verdaderas o falsas (Rosales, 1994, p.16).

P11. Son expresiones $u$ oraciones que pueden ser calificadas como verdaderas (V) o falsas (F); pero no ambas simultáneamente (Chávez, 1997, p.1). 


\section{Otras definiciones:}

P12. Una proposición se define como una aseveración que puede ser verdadera o falsa, pero no ambas (Miller, Heeren \& Hornsby, 2006, p.95).

P13. Una proposición es una frase declarativa que puede ser afirmada o negada (Bustamante, 2009, p.7).

P14. Son aquellas expresiones $u$ oraciones que pueden ser calificadas bien como verdaderas o bien como falsas, sin ambigüedades (Venero, 2012, p.1).

La definición de argumento (razonamiento, deducción o inferencia) hace referencia a premisa y conclusión y estas son definidas como proposiciones. Que los conocimientos que brinda sean claros y precisos y la relación entre ellos está bien establecida es otra de las cosas que refiere Miró Quesada (1978) para una teoría científica. Definir es fijar con claridad, exactitud y precisión la significación de una palabra o la naturaleza de una persona o $\operatorname{cosa}^{8}$. La definición proporciona una descripción formal acerca de algo. "La noción de la cosa descrita debiera existir con claridad en la mente antes de que se haya establecido esa descripción" (Young, 1929, p.174). Oración y frase son expresiones del lenguaje que se estudian a muy temprana edad. Ambas comprenden un conjunto de palabras con sentido, distinguiéndose la oración por expresar un sentido gramatical completo. Para Miró Quesada (1978) “desde el punto de vista de la lógica una proposición es una oración o frase susceptible de ser verdadera o falsa" (p.127). Son frases expresiones como la interrogativa ¿qué hora es?, la exclamativa ¡viva el Perú! y la imperativa ¡deja de gritar! Sin embargo ninguna de ellas es una proposición en tanto no pueden ser calificadas de verdaderas o falsas. Por el contrario la oración “Lima es la capital del Perú" es una proposición ya que declara una verdad.

De las 14 definiciones de proposición presentadas tenemos que seis de ellas se refieren a las proposiciones como enunciados (P2, P3, P4, P6, P8, P9). De estas, cuatro fueron tomadas de textos escolares (P2, P3, P4, P6). Obsérvese que las definiciones P2 y P3 corresponden a los textos del 4 to y 5 to grado de secundaria de una misma editorial. Si bien estas definiciones no se contradicen creemos que no es necesario cambiar su descripción de grado a otro. Lo mismo podríamos decir con respecto a L1, L2 y L3. Existen objetos cuyas definiciones se pueden ir ampliando conforme se avanza en los grados. Basta con citar la ampliación del concepto de número desde los naturales a los reales. Pero consideramos que en este caso no se justifica. La definición P1, tomada del texto de la misma editorial que P2 y P3, es la más confusa a pesar de estar dirigida a estudiantes del 2do grado. En ella no se precisa si una proposición es un enunciado, expresión, oración, frase u otro. Se menciona que tiene un carácter aseverativo ya que afirma o niega algo sobre un sujeto conocido. No explicita a qué se refiere con "sujeto", aunque propone como ejemplo $x<397$ señalando que no se trata de una proposición ya que se desconoce el sujeto. Aquí el sujeto sería la variable $x$. Parece sugerirse que al no conocerse $x$ no puede afirmarse la verdad o falsedad de la expresión dada. Sin embargo la razón no es en sí que no se conozca $x$ sino que no se conozca nada de él. Así la expresión “Si $x>1$, entonces $x^{2}>1$ ", es una proposición verdadera a pesar de que no conozcamos el valor de $x$. En el texto del tercer grado de esta misma editorial, buscando complementar la definición de lógica, menciona que esta "analiza la estructura de las afirmaciones y las expresa simbólicamente. Para ello considera como elementos del lenguaje a las proposiciones, las funciones proposicionales, los operadores lógicos y los cuantificadores" (Grupo Editorial Norma, 2009b, p.12). No se define qué es 
una proposición. Tal vez esperando que esta definición sea tomada del texto del grado anterior que, como señalamos, en nuestra opinión es la más imprecisa.

De las otras ocho definiciones encontramos que cinco de ellas se refieren a las proposiciones como expresiones (P5, P7, P10, P11, P14) y de estas hay dos que también las considera como oraciones (P11, P14). Una definición refiere la proposición como una aseveración (P12) y otra como una frase declarativa (P13). Aquí encontramos que P5 y P6, correspondientes a los textos de segundo y cuarto grado de una misma editorial (diferente a la anterior) defina una proposición haciendo referencia a términos distintos. En un caso se refiere a una expresión y en el otro a enunciado. No podemos decir que estas definiciones se contradicen, pero tampoco consideramos pertinente cambiar su descripción. Una definición destaca de otra por su simpleza y precisión. Vemos que las definiciones dadas por los textos universitarios son más breves (16 palabras en promedio) y precisas que las de los textos escolares (26 palabras en promedio).

Las definiciones revisadas muestran que, aunque en distinto número y con distintas palabras, existe una mayor uniformidad en la definición del término proposición. En ellas se señala la característica principal del término en su relación con la lógica y la matemática, esto es el hecho de ser un enunciado o expresión del lenguaje que puede calificarse como verdadero o falso. Al mismo tiempo encontramos que las definiciones de proposición de los textos de enseñanza superior muestran un mayor nivel de generalización con respecto a las dadas en los textos de secundaria.

\section{DEFINICIÓN DE ENUNCIADO EN LOS TEXTOS}

Definir un término nuevo supone hacer uso de otros términos cuyos significados se tienen claros. La definición P9 "proposición es un enunciado que puede ser verdadero o falso", tal vez por su brevedad, suele ser mencionada por los docentes de matemáticas (y estudiantes) cuando se les pregunta por el significado de proposición. P9 considera la proposición como un enunciado. Esto mismo lo encontramos en las definiciones P2, P3, P4, P6 y P8 que también refieren a enunciado. De aquí que resulta justificado revisar y precisar el significado de enunciado. La definición $\mathrm{P}$, tomada de un texto universitario, define proposición como un enunciado, pero en el texto no se precisa qué se entiende por enunciado. Esto mismo ocurre con el texto del 5to grado de donde se tomó P3. De las seis definiciones que se refieren a proposición como enunciado, cuatro de ellas definen el término enunciado. Lo contrario ocurre en la definición P11 en la cual Chávez (1997) no refiere proposición como enunciado, sin embargo en E5 brinda, de forma muy simple, la noción de este término.

E1. Se llama enunciado a toda frase u oración. No son proposiciones los enunciados que son mandatos, deseos, interrogaciones o exclamaciones (Grupo Editorial Norma, 2009c, p.12).

E2. Un enunciado es toda frase u oración que se utiliza en el lenguaje común. Algunos enunciados son afirmaciones, órdenes, interrogaciones, exclamaciones, etcétera (Curo \& Martínez, 2013, p.13).

E3. Enunciado es toda frase u oración. Algunos enunciados son órdenes, admiraciones e interrogaciones; otros son afirmaciones o negaciones (Editorial Santillana, 2012, p.11).

E4. Enunciado es toda expresión del lenguaje (Doroteo \& Gálvez, 2005, p.12).

E5. Enunciado.- Oración o frase (Chávez, 1997, p.1). 
Nótese que E1, E2, E3 y E5 describen un enunciado como una frase u oración. Esta suele ser la descripción más común para enunciado. Moreno considera la proposición como el significado de un enunciado. "Con la palabra enunciado significamos una expresión lingüística que por tender hacia una realidad adquiere la característica de ser verdadera o falsa" (Moreno, 1971, p.1). Para Moreno, debido a su naturaleza lingüística, los enunciados son cosas materiales más fáciles de manejar, lo que hace más sencillo de conocer su estructura que la de una proposición. Bunge (1980) hace referencia a los nominalistas quienes niegan que haya proposiciones y prefieren hablar de oraciones. Las oraciones, al pertenecer a lenguajes escritos o hablados, tienen existencia concreta. "Casi cualquiera puede pronunciar o escribir una oración en algún lenguaje, pero jamás alguien podrá ver u oír una proposición" (Bunge, 1980, p.65). Debido a que las proposiciones no son objetos concretos los nominalistas no ven la necesidad de las proposiciones y les basta con las oraciones. Miró Quesada (1978), acerca de la distinción que se hacía entre enunciado y proposición, señala que “la mayor parte de los autores consideran que una proposición y un enunciado son exactamente lo mismo: expresiones que describen los hechos" (Miró Quesada, 1978, p.132). Bunge señala que así como la Aritmética nos autoriza a afirmar la existencia de números enteros podemos admitir la existencia de objetos conceptuales como las proposiciones. Esto es consistente con el carácter de ciencia formal que tiene la lógica pura. Las ciencias formales "se ocupan de las formas y relaciones del pensamiento y de las entidades constituidas por el pensamiento con prescindencia de los hechos reales a los que puedan referirse" (Rodríguez, 2002, p.66). Haciendo la distinción entre proposición y las oraciones que la enuncian, Bunge (1980) dice que "la significación de una oración es una proposición" (p.69) donde el significado de una proposición está compuesto de su sentido y sus referentes. Una proposición fuera de contexto carece de significado preciso. Es la mención explícita del contexto lo que permite rastrear los vínculos lógicos de una proposición y determinar su sentido pleno.

Observamos que las definiciones E1, E2, E3 y E5 restringen las proposiciones básicamente al lenguaje natural. Según esta definición expresiones matemáticas como $5>2$ o $\operatorname{sen}\left(30^{\circ}\right)=0.8$ no serían proposiciones, ya que no son frases u oraciones. Sin embargo ellas pueden ser perfectamente calificadas como de verdadera y falsa, respectivamente. De aquí que se requiere ampliar el campo de los enunciados de modo que incluyan expresiones de este tipo. Encontramos en E4 una definición más abierta al mencionar "toda expresión del lenguaje" aunque consideramos que hubiese sido mejor decir "toda expresión de un lenguaje". Al igual que en el lenguaje natural, el lenguaje matemático tiene sintaxis y semántica propia. Estas permiten determinar si una expresión matemática tiene sentido y, a partir de ello, determinar su valor de verdad. En P11 Chávez (1997) parece no querer restringir las proposiciones al campo lingüístico, ya que las define como "expresiones u oraciones" y al poner como ejemplo dos expresiones matemáticas “7 - 1 = $2+9-5$ ” y “2 $+3>5$ ”, proposiciones que le asigna los valores de verdad $V$ y $F$, respectivamente. Esto mismo podría entenderse de la definición dada por Venero (2012) quien, en su definición P14 de proposición, busca distinguir entre "expresiones" y "oraciones" al poner como ejemplo de una proposición verdadera $17-6=11$. En el análisis de una expresión, en tanto esta puede ser considerada como una proposición o no, es importante contar con un contexto preciso que otorgue sentido y referencia a la expresión que se analiza.

Si definimos proposición $(P)$ en términos de enunciado $(E)$ y este como una expresión en un lenguaje (L) dado, lo fundamental es que $E$ tenga significado en $L$, de modo que pueda decirse que $P$ o es 
verdadera o es falsa. Para Russell "solo un entendimiento del lenguaje es necesario para conocer una proposición lógica" (como se citó en Monk, 2013, p.78). Diremos que un enunciado es una expresión, en lenguaje natural o matemático, acerca de una cuestión con sentido propio. Así entendido un enunciado las expresiones " $2+1=5$ " y " $\log (1)=0$ " son proposiciones mientras que " $x+2$ " y " 7 <" no lo son, ya que estas últimas no tienen ningún sentido. La afirmación de que la expresión “ $2+1=5$ ” es una proposición se puede justificar enunciándola en lenguaje natural “dos más uno es igual a cinco". Esta última es una frase con sentido completo que declara algo evidentemente falso. Lo anterior muestra un ejemplo de enunciados distintos que tienen el mismo significado. Así los enunciados "tres es mayor que dos", "three is greater than two" y "3 > 2" tienen el mismo significado. En esta línea hay quienes consideran una proposición como el contenido común de oraciones declarativas sinónimas. Entendiendo por lenguaje un sistema de signos usados por una comunidad para comunicarse en forma oral o escrita, la definición de proposición dada por Piscoya (2007) resulta pertinente: "es toda secuencia finita de signos que con sentido puede ser calificada de verdadera o de falsa" (p. 32). Esta forma general de definir proposición también la encontramos en Wikipedia "una proposición es una cadena de signos expresados en un determinado lenguaje", señalándose además que lo que hace de esta cadena una proposición es que sea interpretable. Bunge (1980) admite que las proposiciones carecen de existencia autónoma y existen solo conceptual o formalmente, pero agrega que si queremos hacer lógica matemática debemos fingir que ellas existen. Afirmar la existencia conceptual de una proposición nos lleva a pensar o fingir que son pensables de manera racional. Sabemos la importancia que esto tiene para la matemática cuya naturaleza es, esencialmente, deductiva.

\section{CONCLUSIÓN}

La matemática es una ciencia que trabaja con entes ideales. Bien sean abstractos o sean interpretaciones de la realidad, estos entes formales requieren ser definidos para su estudio. El cuerpo de conocimientos matemáticos se construye deductivamente a partir de axiomas y definiciones. En la geometría euclidiana tenemos uno de los ejemplos más cercanos de construcción deductiva. Cada texto o curso de matemática es en sí mismo un conjunto de conocimientos que debe ser construido. En una misma rama de las matemáticas los contenidos se ordenan secuencialmente de modo que un tema sea una ampliación del anterior. A su vez, los términos y conceptos de un tema servirán de partida para definir los términos y conceptos del posterior. De aquí la importancia de las definiciones en tanto ellas otorgan significado a los términos y objetos con los que trabajamos en las matemáticas.

Las definiciones permiten describir propiedades de un objeto matemáticoy, dentro del marco teórico correspondiente, la combinación de sus propiedades permite enunciar un conjunto de proposiciones matemáticas. Estas proposiciones pueden ser presentadas como propiedades, lemas, teoremas o corolarios. Muchos teoremas son enunciados en la forma "Si p, entonces q", es decir como una proposición condicional. A diferencia de los axiomas, los teoremas requieren ser demostrados. Demostración es un razonamiento mediante el cual se establece la verdad de una proposición. Esencialmente demostrar consiste en probar. Por lo que demostrar la verdad de una proposición matemática significa probar, usando el razonamiento, que lo que dice la proposición matemática es verdadero. Esto supone el uso de definiciones y otras proposiciones relacionadas presentadas anteriormente. Pero también supone hacer explícitos un conjunto de pasos que, debidamente justificados, permitan mostrar la verdad de 
la proposición matemática. Lo fundamental en la demostración es la forma como justificamos los pasos que seguimos al demostrar. Distintas taxonomías del dominio de la matemática consideran la actividad cognitiva de demostrar como perteneciente a los niveles superiores. Por eso la importancia de comprender el significado de las proposiciones matemáticas.

\section{REFERENCIAS}

Aristóteles (2007). Metafísica. Buenos Aires: Gradifco.

Boisvert, J. (2004). La formación del pensamiento crítico. México D.F.: Fondo de Cultura Económica.

Bunge, M. (1966). La ciencia, su método y su filosofía. Buenos Aires: Editorial Siglo XXI.

Bunge, M. (1980). Epistemología: curso de actualización. Buenos Aires: Editorial Siglo XXI.

Bustamante, A. (2009). Lógica y argumentación. Bogotá: Pearson Prentice Hall.

Chávez, B. (1997). Matemáticas Básicas 1. Lima: Editorial Gómez.

Copi, I. (1964). Introducción a la Lógica. Buenos Aires: EUDEBA.

Coveñas, M. (2012). Matemática 3. Lima: Editorial Coveñas.

Curo, A. \& Martínez, M. (2013). Matemática para administradores. Lima: Universidad Peruana de Ciencias Aplicadas.

Doroteo, F. \& Gálvez, R. (2005). Matemática, 3ro de secundaria. Manual para docentes. Lima: Ediciones El Nocedal.

Editorial Santillana (2009). Matemática 2 secundaria. Lima: Autor.

Editorial Santillana (2012). Matemática 4 secundaria. Lima: Autor.

Grupo Editorial Norma (2009a). Lógica-mente 2. Manual del docente. Lima: Autor.

Grupo Editorial Norma (2009b). Lógica-mente 3. Manual del docente. Lima: Autor.

Grupo Editorial Norma (2009c). Lógica-mente 4. Manual del docente. Lima: Autor.

Grupo Editorial Norma (2009d). Lógica-mente 5. Manual del docente. Lima: Autor.

Katayama, R. (2003). Introducción a la Lógica. Lima: Editorial Universitaria URP.

Miller, C. D., Heeren, V. E. \& Hornsby, J. (2006). Matemática: Razonamiento y aplicaciones (10a ed.). México D.F.: Pearson.

MINEDU (2014). Marco del Sistema Curricular Nacional. Tercera versión para el diálogo. Lima: Autor. Recuperado de https://goo.gl/2VYwVh Miró Quesada, F. (1978). Introducción a la filosofía y lógica. Lima: Editorial Universo.

Monk, R. (2013). ¿Fue Russell un filósofo analítico? En H.J. Glock (Ed.), El surgimiento de la Filosofía Analítica (pp. 71-93). México D.F.: Círculo Ometeotl.

Moreno, A. (1967). ¿Qué es la lógica matemática? Buenos Aires: Editorial Columba.

Moreno, A. (1971) Lógica Matemática. (2ª ed.). Buenos Aires: EUDEBA.

Palacios, R. \& García, O. (1998). Lógica matemática como disciplina curricular. Investigación experimental. Lima: Taller de Artes Impresos.

Piscoya, L. (2007). Lógica General (3a ed.). Lima: Ediciones del Vicerrectorado Académico UNMSM.

Rodríguez, M. (2002). La Ciencia. Lima: Fondo editorial UIGV.

Rosales, D. (1994). Introducción a la Lógica (3a ed.). Lima: AMARU Editores.

Vásquez, A. (2013). Los textos de Lógica general en el nivel universitario en el Perú. Revista de Investigación Educativa, 17(31), 155-172.

Venero, A. (2012). Matemática Básica (2ª ed.). Lima: Ediciones Gemar.

Young, J. W. (1929). Fines, valor y métodos de la enseñanza matemática. Buenos Aires: Editorial Losada.

Zúñiga, J. (2013). Precálculo. Lima: Universidad del Pacífico.

(c) Los autores. Este artículo es publicado por la Revista Digital de Investigación en Docencia Universitaria del Área de Institutional Research and Effectiveness de la Dirección de Aseguramiento de la Calidad, Universidad Peruana de Ciencias Aplicadas. Este es un artículo de acceso abierto, distribuido bajo los términos de la LicenciaCreativeCommons Atribución-Compartirlgual 4.0 Internacional.( http://creativecommons.org/licenses/ by-sa/4.0/l, que permite el uso no comercial, distribución y reproducción en cualquier medio, siempre que la obra original sea debidamente citada. 